\title{
Enfermedades periodontales durante el embarazo y su asociación con parto prematuro y bajo peso al nacimiento. Evidencias y controversias
}

\author{
Periodontal diseases in pregnancy and its association with preterm \\ delivery and low birth weight. Evidence and controversies
}

\author{
ALONSO ROSADO A* \\ PÉREZ GÓIMEZ RIM** \\ CRIADO PÉREZ L*** \\ DUQUE ALONSO $\mathrm{N} * * *$ \\ HERNÁNDEZ MARCOS C*** \\ HERNÁNDEZ MARTÍN M $* * *$ \\ LORENZO RODRÍGUEZ V*** \\ MARTÍN MUÑOZ F*** \\ OTÍN REIXA I***
}

\begin{abstract}
Alonso Rosado A, Pérez Gómez RM, Criado Pérez L, Duque Alonso N, Hernández Marcos C, Hernández Martín M, Lorenzo Rodríguez V, Martín Muñoz F, Otín Reixa I. Enfermedades periodontales durante el embarazo y su asociación con parto prematuro y bajo peso al nacimiento. Evidencias y controversias. Av Periodon Implantol. 2010; 22, 2: 85-89
\end{abstract}

\begin{abstract}
RESUMEN
Aunque el embarazo implica cambios fisiológicos que en general no suponen un riesgo por sí mismos para la salud tanto de la madre como del niño, durante este periodo las infecciones de diversa índole pueden poner en riesgo el curso normal del mismo e incluso comprometer la salud del neonato, siendo los partos prematuros y el bajo peso al nacimiento las patologías que con más frecuencia se asocian con las infecciones. Entre estas infecciones comienza a tener cierta relevancia, desde hace ya algunos años la enfermedad periodontal.

Esta revisión bibliográfica recoge las conclusiones de distintas publicaciones sobre la relación entre enfermedad periodontal, los partos prematuros y el bajo peso al nacimiento, encontrándose resultados variables.
\end{abstract}

PALABRAS CLAVE: Enfermedad periodontal, prematuridad, bajo peso, embarazo.

\section{SUMIMARY}

Pregnancy involves physiological changes which, although not meaning a risk in itself for either the mother's or the baby's health, may lead to the development of new pathologies, including periodontal disease. This bibliographical revision compiles the conclusions drawn by different publications about the relationship between periodontal disease and low-weight preterm births, highlighting variable results.

KEY WORDS: Periodontal disease, preterm birth, low birth weight, pregnancy.

Fecha de recepción: 14 de mayo de 2009.

Fecha de aceptación: 26 de mayo de 2009.

\section{INTRODUCCIÓN}

El aumento de evidencias indica que la concomitancia de las enfermedades periodontales durante el embarazo pueden ser un factor de riesgo para el parto pre- maturo, el bajo peso al nacimiento y otras complicaciones.

Para aclarar los posibles mecanismos de la asociación entre la enfermedad periodontal, el parto prematuro

\footnotetext{
* Doctor en Medicina y Cirugía USAL. Profesor asociado Periodoncia USAL. Máster Periodoncia UCM.

** DUE. Supervisora Ginecología HCU Salamanca. Profesora asociada Escuela Universitaria Enfermería Salamanca.

*** Licenciado en Odontología USAL.
} 
y el bajo peso al nacimiento, hemos realizado una revisión bibliográfica de los posibles efectos producidos por la infección de patógenos periodontales en mujeres embarazadas incluidas las complicaciones durante el embarazo y postnatales.

Aunque hay algunos resultados contradictorios y problemas potenciales en relación a los riesgos incontrolados subyacentes, la mayoría de los estudios clínicos indican una correlación positiva entre la enfermedad periodontal y posibles complicaciones en el embarazo. Estudios recientes también han demostrado que existen factores microbiológicos e inmunológicos que apoyan firmemente esta asociación (1,5,8,9,11-15).

Los nacimientos de bajo peso podrían estar influenciados por el papel de las infecciones maternas $(4,12,16)$. Así, dado que la enfermedad periodontal constituye una infección de los tejidos periodontales en la que existe una elevación local de las prostaglandinas y citocinas proinflamatorias, se ha examinado el riesgo de la relación existente entre la enfermedad periodontal materna y los nacimientos pretérmino. Se han publicado evidencias que indican que más del 18\% de los nacimientos de bajo peso se deberían atribuir a la enfermedad periodontal $(13,14)$. A pesar de ello, no se ha confirmado la asociación entre la enfermedad periodontal y el aumento del riesgo de nacimientos de bajo peso o pretérmino siendo necesaria la realización de otros estudios $(9,19)$. También se ha planteado la posibilidad de que las infecciones periodontales durante el embarazo puedan tener consecuencias negativas a largo plazo sobre el desarrollo infantil $(1,2)$.

Sin embargo, serían necesarios más estudios longitudinales para poder establecer la asociación entre infección periodontal y embarazo de riesgo, y si dicha hipótesis pudiera ser confirmada habría que considerar la enfermedad periodontal como factor de riesgo para el bajo peso al nacimiento y el parto prematuro $(1,3,4)$.

La capacidad de los patógenos periodontales y de sus factores de virulencia para difundirse e inducir tanto a nivel local como sistémico respuestas inflamatorias en el huésped, conduce a la hipótesis de que la enfermedad periodontal puede tener consecuencias más allá de los tejidos periodontales. La inflamación podría difundirse a la unidad fetoplacentaria, a través de la circulación sanguínea. La integridad estructural de la placenta es vital para el normal intercambio de nutrientes entre la madre y el feto, por lo que este daño tisular a la placenta puede contribuir al retraso del crecimiento fetal, con posible bajo peso al nacer. El aumento en la producción de citoquinas inflamatorias y proteína C-reactiva puede contribuir a la contracción uterina y dar lugar a aborto involuntario o parto prematuro y bajo peso al nacimiento $(1,5,6)$.

Las bacterias orales y no solo los patógenos periodontales, también se asocian con efectos adversos durante el embarazo $(7,26,31)$.

Se ha establecido que se da una mayor tasa de partos prematuros entre las madres sin una protección de inmunoglobulina IgG. Por otra parte, la respuesta fetal mediante IgM es más fuerte en los recién nacidos prematuros que en los recién nacidos a término. Estos estudios microbiológicos e inmunológicos en seres humanos proporcionan un fuerte argumento en favor de esta asociación de causalidad $(1,8,31,35)$.

Las hormonas sexuales femeninas desempeñan un papel importante en ese proceso, ya que su concentración aumenta considerablemente en el organismo durante el embarazo. La respuesta de los tejidos a las hormonas sexuales femeninas va a depender del tipo de hormona circulante, de la proporción de estrógeno/progesterona así como de su concentración tisular $(26,27)$. Durante el embarazo aumenta la carga de bacterias aerobias y anaerobias en la cavidad bucal especialmente de Prevotella intermedia, debido a la semejanza estructural de la progesterona y el estradiol con la vitamina $\mathrm{K}$ necesaria para su crecimiento $(9,10,26-28)$.

La combinación de los mediadores de la inflamación junto con el aumento de la concentración hormonal, puede llegar a constituir un factor de riesgo para la integridad de la unidad fetoplacentaria en mujeres con una respuesta inmunitaria celular reducida como consecuencia del embarazo $(9,31,34)$.

\section{CONTROVERSIAS ENTRE LA ENFERMEDAD PERIODONTAL Y LAS COIMPLICACIONES EN EL EMBARAZO}

Diversos estudios han demostrado una asociación entre la enfermedad periodontal y las complicaciones en el embarazo, algunos han llegado a la conclusión de que esta asociación puede estar presente $(1,5,8,11$, $12,15)$, otros en cambio no lo han podido demostrar $(9,19)$. La diversidad de resultados entre estos estudios podría ser explicada por las diferencias en el tamaño 
de las muestras estudiadas, la discriminación racial, las diferencias socioeconómicas poblacionales, o la variabilidad de los criterios para definir la enfermedad periodontal. Algunos estudios utilizan el Community Periodontal Index of Treatment Needs (CPITN), la hemorragia en el sondaje o la profundidad de sondaje de las bolsas periodontales (1).

También se ha de tener en cuenta que no todos los estudios evalúan los mismos resultados; algunos investigan la asociación de la enfermedad periodontal con el bajo peso al nacer, otros la asociación con los partos prematuros $(1,14,17,20,21)$.

En los estudios de Offenbacher y cols., entre otros, se llegó a la conclusión de que había una relación significativa entre la enfermedad periodontal y los partos prematuros y el bajo peso al nacer (1, 5, 8, 9, 1 1-15,32, $33,35)$.

Sin embargo, Moreu y cols. llegaron a la conclusión de que había relación significativa entre la enfermedad periodontal y el bajo peso al nacer, pero no la encontró para el parto prematuro $(4,16,17)$. Por el contrario, Águeda y cols. encontraron asociación entre periodontitis y parto prematuro pero no entre periodontitis y bajo peso al nacer (18).

Por otra parte, Noack y cols., entre otros, llegaron a la conclusión de que la enfermedad periodontal no es un factor de riesgo determinante para el parto prematuro ni para el bajo peso al nacer $(9,19,23,29)$. Aunque si se ha observado en general una tendencia a la asociación, aunque no siempre de forma significativa (9).

Dasanayake y cols. concluyeron que una salud periodontal deficiente en la madre es un factor de riesgo potencial para el parto prematuro y el bajo peso al nacer $(7,20)$.

En otros estudios se pone de manifiesto que el tratamiento y la profilaxis de la enfermedad periodontal reduce el riesgo de parto prematuro y el bajo peso al nacer $(4,21,32)$ y que por tanto es de gran importancia el control de la salud periodontal durante el proceso gestacional (22,32-34).

Por el contrario, otros estudios ponen de manifiesto que no hay pruebas concluyentes de que el tratamiento de la enfermedad periodontal pueda reducir el riesgo de parto prematuro y el bajo peso al nacer, sin embargo, este tratamiento es seguro y mejora la salud periodontal de la madre $(23,24,29,30)$.

\section{CONCLUSIONES}

Debido a que la existencia de asociación entre la enfermedad periodontal y bajo peso al nacer y parto prematuro es aún motivo de controversia, se ha realizado esta revisión bibliográfica con el objetivo de poder llegar a una conclusión que pueda ayudar a disminuir dichas complicaciones.

Como hemos visto existen discrepancias en los resultados obtenidos en los diferentes estudios, y aunque la mayoría coinciden en que existe una relación entre estas complicaciones del embarazo y la enfermedad periodontal, $(1,5,8,12-14$,$) hemos de tener en cuenta$ aquellos estudios que establecen que dicha relación no es significativa $(9,19,29,30)$.

Es importante señalar que todos los estudios realizados hasta la fecha que han llevado cabo un tratamiento periodontal en mujeres embarazadas (generalmente en el segundo trimestre del embarazo) sugieren que este tratamiento es seguro para la madre y el niño. Por lo tanto, se puede proporcionar tratamiento periodontal con seguridad durante el embarazo para mejorar la salud bucal de la madre $(4,21,22,25,32)$. Sin embargo se desconoce si este tratamiento también mejora significativamente el resultado del embarazo $(23,24)$.

Dado que la enfermedad periodontal se puede prevenir y tratar, debe ser el odontólogo el que tenga la responsabilidad de diagnosticar y tratar adecuadamente a las mujeres que están embarazadas o que planean quedarse embarazadas. También es responsabilidad del odontólogo informar a los pacientes de que si no se trata la enfermedad periodontal puede aumentar el riesgo no sólo de sufrir complicaciones en el embarazo, sino también de desarrollar condiciones desfavorables que pueden afectar al bienestar de los bebés. No hay evidencias en contra de la prestación de estos cuidados a las madres, lo que sugiere que este tratamiento realmente puede ser beneficioso para ambos. Así mismo, algunos estudios plantean la posibilidad de que en el futuro, la inmunización materna puede ayudar a proporcionar protección al feto durante el embarazo $(1,31,35)$.

Existe un alto porcentaje de mujeres con enfermedad periodontal en el embarazo, por lo que son muchas las que se beneficiarían directamente del cuidado prenatal $(4,13,14,21,22,32)$.

Desde nuestro punto de vista y tras analizar la literatura, creemos firmemente que es recomendable que las mujeres embarazadas tengan un especial cuidado con 
la salud bucodental (visitas al odontólogo, higiene oral adecuada, y tratamientos periodontales si son necesarios) $(4,21,22,25,32)$.

\section{BIBLIOGRAFÍA}

1. Bobetsis YA, Barros SP, Offenbacher S. Exploring the relationship between periodontal disease and pregnancy complications. J Am Dent Assoc 2006;2(137):7S-13S.

2. Davenport ES, Williams CE, Sterne JA, et al. Maternal periodontal disease and preterm low birthweight: casecontrol study. J Dent Res 2002;81(5): 313-8.

3. Romero BC, Chiquito CS, Elejalde LE, Bernardoni CB. Relationship Between Periodontal Disease in Pregnant Women and the Nutritional Condition of Their Newborns. J Periodontol 2002;10(73):1177-83.

4. Xiong X, Buekens P, Fraser WD, Beck J, Offenbacher S. Periodontal disease and adverse pregnancy outcomes: a systematic review. BJOG 2006;113(2):135-43.

5. López NJ, Smith PC, Gutiérrez J. Higher risk of preterm birth and low birth weight in women with periodontal disease. J Dent Res 2002;81(1):58-63.

6. Pitiphat W, Joshipura KJ, Rich-Edwards JW, Williams PL, Douglass CW, Gillman MW. Periodontitis and Plasma CReactive Protein During Pregnancy. J Periodontol 2006; 5(77):821-5.

7. Dasanayake AP, Li Y, Wiener H, Ruby JD, Lee MJ. Salivary Actinomyces naeslundii Genospecies 2 and Lactobacillus casei Levels Predict Pregnancy Outcomes. J Periodontol 2005;2(76):171-7.

8. Robles JJ, Salazar F, Proaño D. Enfermedad periodontal como factor de riesgo del retardo intrauterino. Rev Estomatol Herediana 2004;1-2(14):27-34.

9. Castaldi JL, Bertin MS, Gimenez F, Lede R. Periodontal disease: Is it a risk factor for premature labor, low birth weight or preeclampsia? Rev Panam Salud Publica 2006; 19(4):253-8.

10. Kornman KS, Loesche WJ. The subgingival microbial flora during pregnancy.J Periodontal Res. 1980 Mar;15(2):111-22.

11. Dortbudak O, Eberhardt R, Ulm M, Persson GR. Periodontitis, A marker of risk in pregnancy for preterm birth. J Clin Periodontol 2005;32:45-52.
12. Alves, RT, Ribeiro RA. Relationship between maternal periodontal disease and birth of preterm low weight babies. Braz. Oral Res 2006;20(4):318-23.

13. Offenbacher S, Lieff S, Boggess KA, Murtha AP, Madianos PN, Champagne CME, et al. Maternal Periodontitis and Prematurity. Part I: Obstetric Outcome of Prematurity and Growth Restriction. Annals of Periodontology 2001; $1(6): 164-74$

14. Offenbacher S, Boggess KA, Murtha AP, Jared HL, Lieff S, McKaig RG. Progressive Periodontal Disease and Risk of Very Preterm Delivery. Obstetrics \& Gynecology 2006; 107:29-36.

15. Lieff S, Boggess KA, Murtha AP, Jared H, Madianos PN, Moss K, et al.The Oral Conditions and Pregnancy Study: Periodontal Status of a Cohort of Pregnant Women. J Periodontol 2004;1(75):116-26.

16. Moreu G, Téllez L, González-Jaranay M. Relationship between maternal periodontal disease and low-birthweight pre-term infants. Journal of Clinical Periodontology $2005 ; 32(6): 622-7$.

17. Jeffcoat MK, Geurs NC, Reddy MS, Cliver SP, Goldenerg $\mathrm{RL}$, Hauth JC. Periodontal infection and preterm birth: results of a prospective study. Journal of the Americal Dental Association 2001;132:875-80.

18. Agueda A, Ramón JM, Manau C, Guerrero A, Echeverría JJ. Periodontal disease as a risk factor for adverse pregnancy outcome: a prospective cohort study. J Clin Periodontol 2008 Jan;35(1):16-22.

19. Noack B, Klingenberg J, Weigelt J, Hoffmann T. Periodontal status and preterm low birth weight: a case control study. J Periodontal Res 2005;40(4):339-45.

20. Dasanayake AP. Poor periodontal health of the pregnant woman as a risk factor for low birth weight. Ann Periodontol. 1998;3(1): 206-12.

21. Jeffcoat MK, Hauth, JC, Geurs NC, Reddy MS, Cliver SP, Hodgkins PM, Goldenberg RL. Periodontal disease and preterm birth. Results of a pilot intervention study. Journal of Periodontology 2003;74:1214-8.

22. Coutinho NN, Rocha ES, Ferreira RC, Vilaca EL, Moreira AN, Magalhaes CS. Control de la enfermedad periodontal y caries en gestantes. Rev. Fundac. Juan Jose Carraro 2005;10(21):33-9. 
23. Michalowicz BS, Hodges JS, DiAngelis AJ, Lupo VR, Novak MJ, Ferguson JE, et al. Treatment of periodontal disease and the risk of preterm birth. N Engl J Med 2006, 2;355 (18):1885-94.

24. Khader YS, Ta'ani Q. Periodontal Diseases and the Risk of Preterm Birth and Low Birth Weight: A Meta-Analysis. J Periodontol 2005;2(76):161-165.

25. Novak MJ, Novak KF, Hodges JS, Kirakodu S, Govindaswami M, Diangelis A, Buchanan W, Papapanou PN, Michalowicz BS.:Periodontal bacterial profiles in pregnant women: response to treatment and associations with birth outcomes in the obstetrics and periodontal therapy (OPT) study. J Periodontol 2008 Oct;79(10): 1870-9.

26. Lopatin DE, Kornman KS, Loesche WJ.: Modulation of immunoreactivity to periodontal disease-associated microorganisms during pregnancy. Infect Immun. 1980 Jun;28(3):713-8.

27. Raber-Durlacher JE, van Steenbergen TJ, Van der Velden U, de Graaff J, Abraham-Inpijn L. Experimental gingivitis during pregnancy and post-partum: clinical, endocrinological, and microbiological aspects.J Clin Periodontol. 1994 Sep;21(8):549-58.

28. Ojanotko-Harri AO, Harri MP, Hurttia HM, Sewón LA. Altered tissue metabolism of progesterone in pregnancy gingivitis and granuloma. J Clin Periodontol 1991 Apr;18(4):262-6

29. Michalowicz BS, DiAngelis AJ, Novak MJ, Buchanan W, Papapanou PN, Mitchell DA, Curran AE, Lupo VR,
Ferguson JE, Bofill J, Matseoane S, Deinard AS Jr, Rogers TB. Examining the safety of dental treatment in pregnant women J Am Dent Assoc 2008 Jun; 139(6):685-95.

30. Castaldi JL, Bertin MS, Giménez F, Lede R. Periodontal disease: Is it a risk factor for premature labor, low birth weight or preeclampsia? Rev Panam Salud Publica. 2006 Apr;19(4):253-8.

31. Horton AL, Boggess KA, Moss KL, Jared HL, Beck J, Offenbacher S J. Periodontal disease early in pregnancy is associated with maternal systemic inflammation among African American women. Periodontol 2008 Jul;79(7):1127-32.

32. Bobetsis YA, Barros SP, Offenbacher S.: Exploring the relationship between periodontal disease and pregnancy complications.J Am Dent Assoc 2006 Oct; 137 Suppl:7S$13 \mathrm{~S}$.

33. Xiong X, Buekens P, Fraser WD, Beck J, Offenbacher S. Periodontal disease and adverse pregnancy outcomes: a systematic review. BJOG 2006 Feb; 113(2):135-43.

34. Boggess KA, Lieff S, Murtha AP, Moss K, Jared H, Beck J, Offenbacher S.: Maternal serum C-reactive protein concentration early in pregnancy and subsequent pregnancy loss. Am J Perinatol 2005 Aug;22(6):299304.

35. Boggess KA, Moss K, Madianos P, Murtha AP, Beck J, Offenbacher S. Fetal immune response to oral pathogens and risk of preterm birth. Am J Obstet Gynecol 2005 Sep;193(3 Pt 2):1121-6. 